\title{
Instrumentation of a Level-1 Track Trigger in the ATLAS detector for the High Luminosity LHC
}

\section{Royal Holloway
University of London}

Introduction

- HL-LHC in 2020: L=5x1034 $\mathrm{cm}^{-2} \mathrm{~s}^{-1}$ and $3 a b^{-1}$ of data per experiment - Need lepton triggers at thresholds of $\mathrm{p}_{\text {T }} 25 \mathrm{GeV}$

- But event rates: x 5 and number of interactions per bunch crossing: $<\mu>\sim 120$

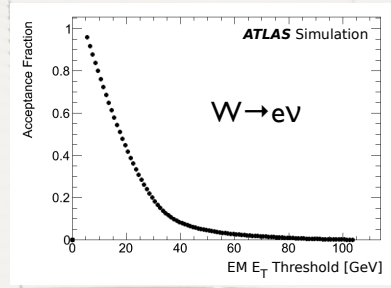

\section{Dr Véronique Boisvert, Royal Holloway, University of London on behalf of the ATLAS Collaboration

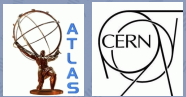

\section{Current ATLAS L1 Trigger and upgrades}

- Hardware L1: $40 \mathrm{MHz}$ to $75 \mathrm{kHz}$, software L2 (Rol scheme): 3kHz, Event Filter: output rate: $\sim 300 \mathrm{~Hz}$

- L1: Coarse calorimeter and muon information, latency $<2.5 \mu \mathrm{s}$

- Want to keep single lepton trigger rate below $20 \mathrm{kHz}$

- During the long shut down of $\sim 2018$ :

- complete replacement of Inner Detector tracker

- replace almost all of the on-detector FE electronics

- FE not replaced: muon drift tube chambers $\rightarrow$ L1A $\sim 200 \mathrm{kHz}$ and latency $\sim 20 \mu \mathrm{s}$

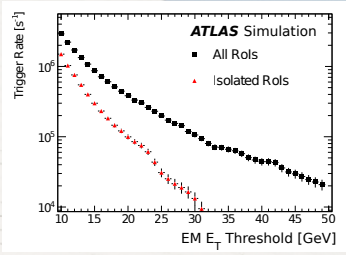

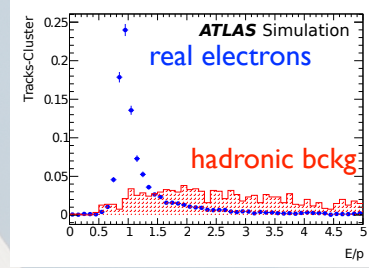

Benefits of Tracking at L1

- Reduce L1A rate (eg current HLT)

- Ensure objects are from Primary

Vertex

- Possible track based isolation

- Added flexibility to trigger system

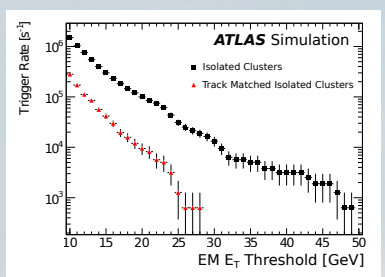

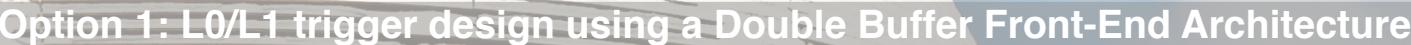

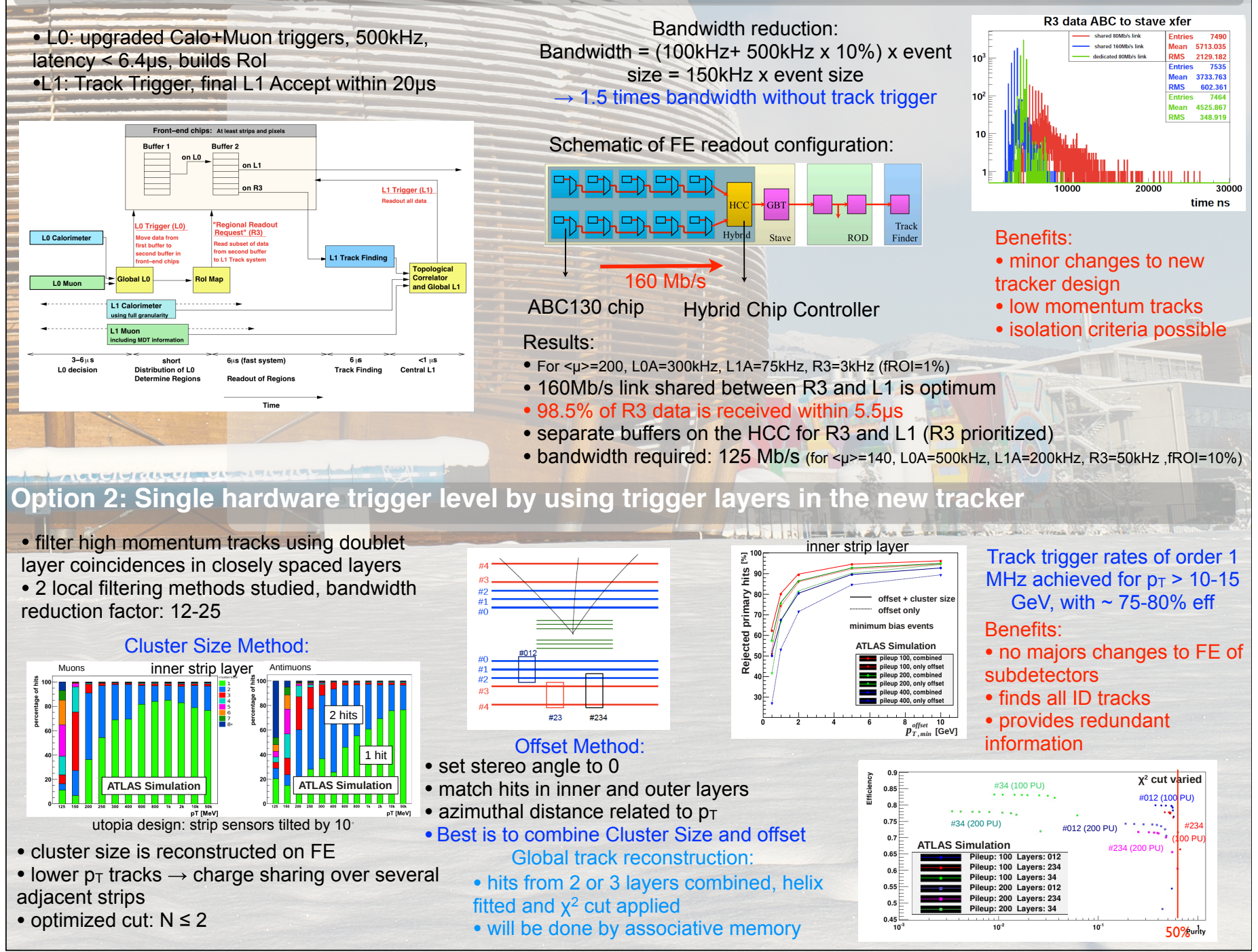

